\title{
Non-syndromic occurrence of true generalized microdontia with mandibular mesiodens - a rare case
}

\author{
Seema D Bargale* and Shital DP Kiran
}

\begin{abstract}
Abnormalities in size of teeth and number of teeth are occasionally recorded in clinical cases. True generalized microdontia is rare case in which all the teeth are smaller than normal. Mesiodens is commonly located in maxilary central incisor region and uncommon in the mandible. In the present case a 12 year-old boy was healthy; normal in appearance and the medical history was noncontributory. The patient was examined and found to have permanent teeth that were smaller than those of the average adult teeth. The true generalized microdontia was accompanied by mandibular mesiodens. This is a unique case report of non-syndromic association of mandibular hyperdontia with true generalized microdontia.
\end{abstract}

Keywords: Generalised microdontia, Hyperdontia, Permanent dentition, Mandibular supernumerary tooth

\section{Introduction}

Microdontia is a rare phenomenon. The term microdontia (microdentism, microdontism) is defined as the condition of having abnormally small teeth [1]. According to Boyle, "in general microdontia, the teeth are small, the crowns short, and normal contact areas between the teeth are frequently missing" [2] Shafer, Hine, and Levy [3] divided microdontia into three types: (1) Microdontia involving only a single tooth; (2) relative generalized microdontia due to relatively small teeth in large jaws and (3) true generalized microdontia, in which all the teeth are smaller than normal. According to these authors, aside from its occurrence in some cases of pituitary dwarfism, true generalized microdontia is exceedingly rare. Microdontia of a single tooth can be further classified into (1) microdontia of the whole tooth, (2) microdontia of the crown of the tooth, and (3) microdontia of the root alone [4].

Involvement of the entire dentition is rare and been reported in radiation or chemotherapeutic treatment during the developmental stage of the teeth [5], pituitary dwarfism [3] and Fanconi's anemia [6]. The syndromes associated with microdontia are Gorlin-Chaudhry-Moss syndrome, Williams's syndrome, Chromosome d/u, 45X

\footnotetext{
* Correspondence: drseemabargale@gmail.com
Department of Pedodontics and Preventive Dentistry, AECS Maruthi Dental

* Correspondence: drseemabargale@gmail.com College and Research Center, India
}

(C) 2011 Bargale and Kiran; licensee BioMed Central Ltd. This is an Open Access article distributed under the terms of the Creative Commons Attribution License (http://creativecommons.org/licenses/by/2.0), which permits unrestricted use, distribution, and reproduction in any medium, provided the original work is properly cited.
[Ullrich-Turner syndrome], Chromosome 13[trisomy 13], Rothmund-Thomson syndrome, Hallermann-Streiff, Orofaciodigital syndrome (type 3), Oculo-mandibulo-facial syndrome, Tricho-Rhino-Phalangeal, type1 Branchiooculo-facial syndrome.

Supernumerary teeth are defined as any supplementary tooth or tooth substance in addition to usual configuration of twenty deciduous and thirty two permanent teeth [7]. Classification of supernumerary teeth may be based on position or morphology. Positional variations include anterior mesiodens, para-premolars, para-molars and distomolars. Variations in morphology consist of supplemental and rudimentary types [8].

Supernumerary teeth are common in the maxillary anterior region although supernumerary teeth have been reported in the incisor region of the mandible are very rare. Although supernumerary teeth have been reported in the incisor region of the mandible, they are very rare [9-14].

Conditions, in which supernumery teeth found, are cleidocranial dysplesia, cleft lip and cleft palate [15]. Syndromes associated with supernumery teeth are Familial adenomatous polyposis [Gardner's], Apert, Klippel-Trenaunay-Weber, Craniometaphyseal dysplasia, Trisomy 21 [Down's], Nance-Horan, Orofaciodigital syndrome (type 3), Sturge-weber and Tricho-Rhino-Phalangeal, type1. 
In the case described here is a bizarre generalized microdontia involving the entire dentition along with mandibular mesiodens without any other apparent systemic conditions.

\section{Case Report}

The patient was a 12 year old boy, only child of consanguineous parents, reported to the department of pedodontics and preventive dentistry with the complaint of small teeth. Parents noted small teeth ever since the eruption of permanent teeth. No abnormalities were reported, however, in their extended family.

\section{Physical examination}

Physical growth was within normal limits. The patient was of normal in stature, appearance, height, and weight for his age. Upon examination of the limbs, hands, skin, hair, nails and eyes were all appeared normal. No abnormality was noted in neck, back, muscles, cranium and joints as well. Intellectual and scholastic performance was also normal. His medical history was unremarkable; no other abnormalities were noted in the history apart from the difficult delivery. The child was examined and found to be free of any gross abnormalities.

His blood profile was normal. Serum calcium, phosphorous and alkaline phosphatase levels were also normal. Endocrinological investigation was carried out to rule in or out the possibility of hormonal disorder, and the results were within normal limits.

\section{Intraoral examination}

The intraoral soft tissues were healthy, but the teeth were abnormal in size and shape (Figure 1 and 2). Diagnostic casts were obtained to aid in diagnosis (Figure 3). Patient was in permanent dentition, teeth present were small in size. The patient had normal occlusion with excessive spacing between the teeth. Fully erupted mandibular mesiodens was present between the central incisors.

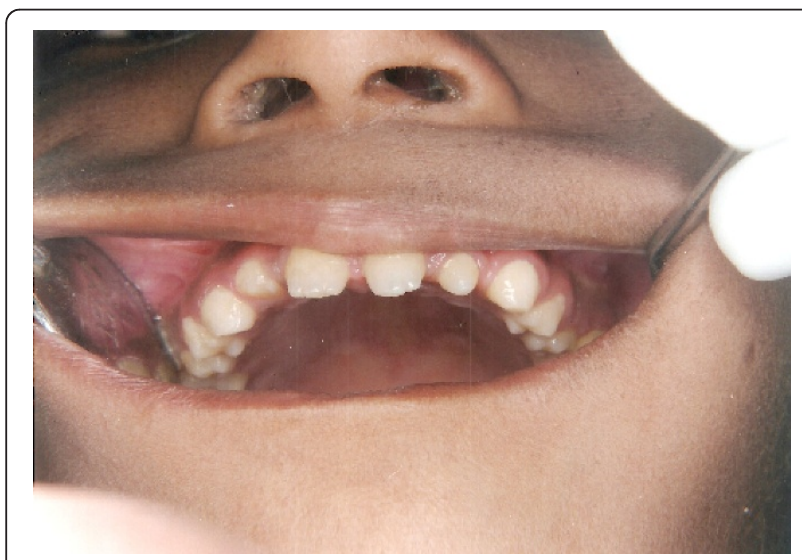

Figure 1 Intra oral view of the upper arch.

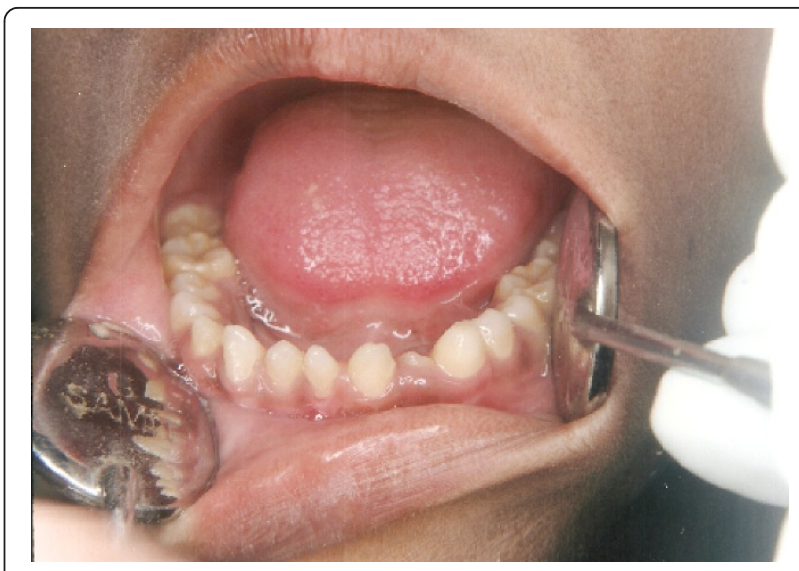

Figure 2 Intra oral view of the lower arch

The anterior teeth lacked normal size in all dimensions. Most of the anterior teeth were "peg-shaped" without the typical variation in mesiodistal and labiolingual dimensions. Almost all the maxillary anterior teeth did not have lingual pits whereas mandibular central and lateral incisors had prominent pits on the lingual surfaces. The posterior teeth were also small and exhibited a short occlusogingival dimension. Overall, the dentition was smaller than that of the average adult (Table 1 and 2). Orthopantomogram or the Intra oral periapical radigraph could not be taken because the patient was not able to afford.

The simultaneous presence of microdontia and supernumery teeth is been reported in the Cleidocranial dysplasia, Craniometadiaphyseal dysplasia, Dermoodontodysplasia, Hypodontia and nail dysgenesis, Orofaciodigital syndrome type 3 and Tricho-rhino-phalangeal syndrome type 1 . However in this case, except for the dental abnormality in the form of generalized microdontia and the presence of fully erupted mandibular mesiodens between the central incisors were found and no other clinical features observed, therefore all the syndrome associated with the simultaneous presence of microdontia and supernumery teeth were ruled out along with Taurodontism, microdontia, and dens invaginatus as well as Distal symphalangism,

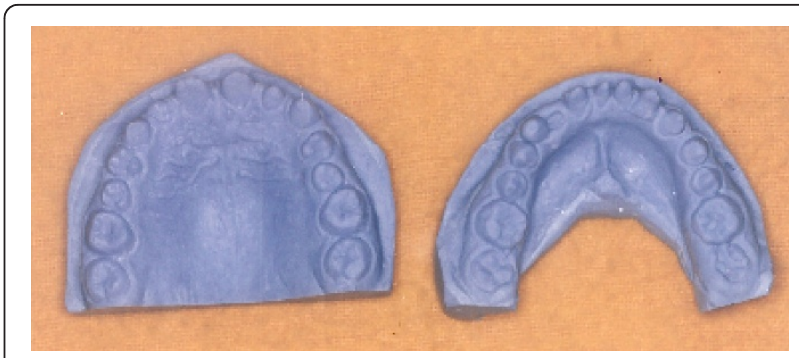

Figure 3 Diagnostic casts showing the morphology of the teeth. 
Table 1 Comparison of buccolingual/labiolingual and mesiodistal crown dimensions with an anatomic average* of the right side maxillary and mandibular teeth

\begin{tabular}{|c|c|c|c|c|c|c|c|c|}
\hline Right side & Central incisor & Lateral incisor & Canine & First premolar & Second premolar & First molar & Second molar & Total \\
\hline Maxillary & $\frac{\mathrm{MD}}{\mathrm{LL}}$ & $\frac{\mathrm{MD}}{\mathrm{LL}}$ & $\frac{\mathrm{MD}}{\mathrm{LL}}$ & $\frac{\mathrm{MD}}{\mathrm{BL}}$ & $\frac{\mathrm{MD}}{\mathrm{BL}}$ & $\frac{\mathrm{MD}}{\mathrm{BL}}$ & $\frac{\mathrm{MD}}{\mathrm{BL}}$ & $\frac{\mathrm{MD}}{\mathrm{LL} / \mathrm{BL}}$ \\
\hline Average & $\frac{8.5}{7.0}$ & $\frac{6.5}{6.0}$ & $\frac{7.5}{8.0}$ & $\frac{7.0}{9.0}$ & $\frac{7.0}{9.0}$ & $\frac{10.0}{11.0}$ & $\frac{9.0}{11.0}$ & $\frac{55.5}{61.0}$ \\
\hline Patient & $\frac{7.5}{6.1}$ & $\frac{4.7}{5.6}$ & $\frac{6.9}{7.4}$ & $\frac{6.4}{7.8}$ & $\frac{6.5}{7.7}$ & $\frac{9.5}{10.4}$ & $\frac{8.7}{10.9}$ & $\frac{50.2}{55.9}$ \\
\hline Mandible & $\frac{\mathrm{MD}}{\mathrm{LL}}$ & $\frac{\mathrm{MD}}{\mathrm{LL}}$ & $\frac{\mathrm{MD}}{\mathrm{LL}}$ & $\frac{\mathrm{MD}}{\mathrm{BL}}$ & $\frac{\mathrm{MD}}{\mathrm{BL}}$ & $\frac{\mathrm{MD}}{\mathrm{BL}}$ & $\frac{\mathrm{MD}}{\mathrm{BL}}$ & $\frac{\mathrm{MD}}{\mathrm{LL} / \mathrm{BL}}$ \\
\hline Average & $\frac{5.0}{6.0}$ & $\frac{5.5}{6.5}$ & $\frac{7.0}{7.5}$ & $\frac{7.0}{7.5}$ & $\frac{7.0}{8.0}$ & $\frac{11.0}{10.5}$ & $\frac{10.5}{10.0}$ & $\frac{53.0}{56.0}$ \\
\hline Patient & $\frac{4.4}{5.6}$ & $\frac{5.2}{5.8}$ & $\frac{6.6}{7.1}$ & $\frac{6.7}{7.3}$ & $\frac{6.8}{7.7}$ & $\frac{10.7}{10.3}$ & $\frac{9.9}{9.4}$ & $\frac{50.3}{53.2}$ \\
\hline
\end{tabular}

Measurements in millimeters were taken at widest portion of clinical crown on diagnostic casts. ${ }^{*}$ Anatomic average taken from Wheeler, R. C.: Textbook of Dental Anatomy and Physiology, ed. 7, Philadelphia, 1993, W. B. Saunders Company, pp. 25.

hypoplastic carpal bones, microdontia, dental pulp stones, narrowed zygomatic arch (Table 3).

A diagnosis of non-syndromic occurance of true generalized microdontia with mandibular mesiodens was made as no systemic condition was observed. The fully erupted mandibular mesiodens was extracted under local anesthesia in order to correct midline and to facilitate the orthodontic treatment.

\section{Discussion}

The initiating factor or factors responsible for microdontia remain obscure. Mutation in developmental regularity genes are known to cause variety of dental defects [16]. Both genetic and environmental factors are involved in the complex etiology of microdontia. Genetic factors probably play a role in the formation of microdontia. Although the proband was the only child, the presence of consanguinity in the form of both parents being maternal first cousins could suggest recessive or polygenic inheritance.
The development of a tooth has been shown to have ectodermal, mesodermal, and neural crest contributions. The variation in size of a particular tooth arises during the period when the form of the tooth is being determined by the enamel organ and the sheath of hertwig at the bell stage of enamel organ. The determination of the form of the crown is thought to be related to different regions of the oral epithelium or to the ectomesenchyme. Studies have shown that different regions of the oral epithelium rather than the underlying ectomesenchyme are initially responsible for the shape of the crown [17]. Bones dating from the Middle Ages which were excavated at Alborg, Denmark proved evidence for generalized microdontia resulting from intrauterine growth retardation [18].

On the basis of visual documentation, the patient in the current case seems to have been more severely affected in all his teeth which exhibited aberrant morphology and all were smaller than normal. MEDLINE search in the English dental literature for true generalized microdontia revealed zero search results. Although child's mother had

Table 2 Comparison of buccolingual/labiolingual and mesiodistal crown dimensions with an anatomic average* of the left side maxillary and mandibular teeth

\begin{tabular}{lllllllll}
\hline Left side & Central incisor & Lateral incisor & Canine & First premolar & Second premolar & First molar & Second molar & Total \\
\hline Maxillary & $\mathrm{MD}$ & $\frac{\mathrm{MD}}{\mathrm{LL}}$ & $\frac{\mathrm{ML}}{\mathrm{LL}}$ & $\frac{\mathrm{MD}}{\mathrm{BL}}$ & $\frac{\mathrm{MD}}{\mathrm{BL}}$ & $\frac{\mathrm{MD}}{\mathrm{BL}}$ & $\frac{\mathrm{MD}}{\mathrm{BL}}$ & $\frac{\mathrm{MD}}{\mathrm{LL} / \mathrm{BL}}$ \\
\hline Average & $\frac{8.5}{7.0}$ & $\frac{6.5}{6.0}$ & $\frac{7.5}{8.0}$ & $\frac{7.0}{9.0}$ & $\frac{7.0}{9.0}$ & $\frac{10.0}{11.0}$ & $\frac{9.0}{11.0}$ & $\frac{55.5}{61.0}$ \\
\hline Patient & $\frac{7.2}{5.8}$ & $\frac{4.5}{5.4}$ & $\frac{7.1}{7.5}$ & $\frac{6.2}{7.6}$ & $\frac{6.3}{7.7}$ & $\frac{9.7}{10.9}$ & $\frac{49.3}{52.1}$ \\
\hline Mandible & $\frac{\mathrm{MD}}{\mathrm{LL}}$ & $\frac{\mathrm{MD}}{\mathrm{LL}}$ & $\frac{\mathrm{MD}}{\mathrm{LL}}$ & $\frac{\mathrm{MD}}{\mathrm{BL}}$ & $\frac{\mathrm{MD}}{\mathrm{BL}}$ & $\frac{11.0}{\mathrm{BL}}$ & $\frac{10.5}{10.0}$ & $\frac{\mathrm{MD}}{\mathrm{LL} / \mathrm{BL}}$ \\
\hline Average & $\frac{5.0}{6.0}$ & $\frac{5.5}{6.5}$ & $\frac{7.0}{7.5}$ & $\frac{7.0}{7.5}$ & $\frac{7.0}{8.0}$ & $\frac{10.7}{10.2}$ & $\frac{9.6}{8.9}$ & $\frac{53.0}{56.0}$ \\
\hline Patient & $\frac{4.2}{5.7}$ & $\frac{5.3}{6.1}$ & $\frac{6.7}{7.3}$ & $\frac{6.7}{7.2}$ & $\frac{6.8}{7.7}$ & $\frac{50.0}{53.1}$ \\
\hline
\end{tabular}

Measurements in millimeters were taken at widest portion of clinical crown on diagnostic casts. *Anatomic average taken from Wheeler, R. C.: Textbook of Dental Anatomy and Physiology, ed. 7, Philadelphia, 1993, W. B. Saunders Company, pp. 25. 
Table 3 Comparison of conditions associated with the simultaneous presence of microdontia and supernumery teeth along with taurodontism, microdontia, and dens invaginatus as well as distal symphalangism, hypoplastic carpal bones, microdontia, dental pulp stones, narrowed zygomatic arch.

\begin{tabular}{|c|c|c|c|c|c|c|c|}
\hline $\begin{array}{l}\text { Taurodontism, } \\
\text { microdontia, and } \\
\text { dens invaginatus }\end{array}$ & $\begin{array}{l}\text { Cleidocranial } \\
\text { dysplasia }\end{array}$ & $\begin{array}{l}\text { Craniometadiaphyseal } \\
\text { dysplasia }\end{array}$ & Dermoodontodysplasia & $\begin{array}{l}\text { Hypodontia and } \\
\text { nail dysgenesis }\end{array}$ & $\begin{array}{l}\text { Orofaciodigital } \\
\text { syndrome type } 3\end{array}$ & $\begin{array}{l}\text { Tricho-rhino- } \\
\text { phalangeal } \\
\text { syndrome type } \\
1\end{array}$ & $\begin{array}{l}\text { Distal symphalangism, hypoplastic } \\
\text { carpal bones, microdontia, dental pulp } \\
\text { stones, narrowed zygomatic arch }\end{array}$ \\
\hline $\begin{array}{l}\text { Generalized } \\
\text { microdontia }\end{array}$ & $\begin{array}{l}\text { Autosomal } \\
\text { dominant }\end{array}$ & Autosomal recessive & Autosomal dominant & $\begin{array}{l}\text { Autosomal } \\
\text { dominant }\end{array}$ & $\begin{array}{l}\text { Autosomal } \\
\text { recessive }\end{array}$ & $\begin{array}{l}\text { Autosomal } \\
\text { dominant }\end{array}$ & Autosomal dominant \\
\hline $\begin{array}{l}\text { Taurodontism of } \\
\text { first permanent } \\
\text { molars }\end{array}$ & Chromosome 6 & Macrocephaly & Dry skin & Chromosome 4 & $\begin{array}{l}\text { Thin/hyperconvex/ } \\
\text { hypoplastic nails }\end{array}$ & $\begin{array}{l}\text { Autosomal } \\
\text { recessive }\end{array}$ & Absent/small nails \\
\hline $\begin{array}{l}\text { Multiple teeth with } \\
\text { one or more dens } \\
\text { invaginatus }\end{array}$ & Arm $p$ & Frontal bossing & Ichthyosis & Arm $p$ & Prominent occiput & Chromosome 8 & Microdontia \\
\hline \multirow[t]{11}{*}{$\begin{array}{l}\text { X-linked recessive } \\
\text { inheritance }\end{array}$} & $\begin{array}{l}\text { Normal height } \\
\text { (with skeletal } \\
\text { dysplasia) }\end{array}$ & Large fontanelle & Thin skin & Dry skin & Frontal bossing & Arm q & Other dental abnormality \\
\hline & $\begin{array}{l}\text { Short stature - } \\
\text { postnatal }\end{array}$ & Prominent eyes & Pigmented naevi & Fine hair & Round face & $\begin{array}{l}\text { Normal height } \\
\text { (with skeletal } \\
\text { dysplasia) }\end{array}$ & Abnormal clinical features of the limbs \\
\hline & Absent/small nails & $\begin{array}{l}\text { Mandibular } \\
\text { hyperostosis/sclerosis }\end{array}$ & Abnormal hair texture & $\begin{array}{l}\text { Brittle hair/ } \\
\text { trichorrhexis } \\
\text { nodosa/pili torti }\end{array}$ & Hypertelorism & $\begin{array}{l}\text { Short stature - } \\
\text { postnatal }\end{array}$ & Brachydactyly \\
\hline & Macrocephaly & $\begin{array}{l}\text { Optic nerve } \\
\text { abnormality/atrophy }\end{array}$ & $\begin{array}{l}\text { Sparse/absent scalp hair } \\
\text { - localised }\end{array}$ & $\begin{array}{l}\text { Sparse/absent } \\
\text { scalp hair - } \\
\text { generalised }\end{array}$ & $\begin{array}{l}\text { Down-slanting } \\
\text { palpebral fissures }\end{array}$ & $\begin{array}{l}\text { Decreased body } \\
\text { hair/hypotrichosis }\end{array}$ & Irregularities of length/shape of fingers \\
\hline & $\begin{array}{l}\text { Flat occiput } \\
\text { (brachycephaly) }\end{array}$ & Microdontia & Abnormal nails & Absent/small nails & $\begin{array}{l}\text { Other orbital } \\
\text { abnormality }\end{array}$ & $\begin{array}{l}\text { Decreased hair } \\
\text { pigmentation - } \\
\text { general }\end{array}$ & Syndactyly of fingers \\
\hline & Frontal bossing & $\begin{array}{l}\text { Abnormal tooth } \\
\text { position/malocclusion/ } \\
\text { open bite }\end{array}$ & $\begin{array}{l}\text { Midface hypoplasia/flat/ } \\
\text { short midface }\end{array}$ & $\begin{array}{l}\text { Thin/ } \\
\text { hyperconvex/ } \\
\text { hypoplastic nails }\end{array}$ & $\begin{array}{l}\text { Paresis of ocular } \\
\text { muscles/squint }\end{array}$ & $\begin{array}{l}\text { Decreased hair } \\
\text { pigmentation - } \\
\text { patchy }\end{array}$ & Short foot (including brachydactyly) \\
\hline & $\begin{array}{l}\text { Wide sutures/ } \\
\text { delayed fusion of } \\
\text { sutures }\end{array}$ & $\begin{array}{l}\text { Missing permanent } \\
\text { teeth/retained } \\
\text { deciduous teeth }\end{array}$ & $\begin{array}{l}\text { Micrognathia/agnathia/ } \\
\text { retrognathia }\end{array}$ & $\begin{array}{l}\text { Dysplastic/ } \\
\text { grooved/thick/ } \\
\text { discoloured nails }\end{array}$ & $\begin{array}{l}\text { Other eye } \\
\text { movement } \\
\text { disorder }\end{array}$ & Fine hair & $\begin{array}{l}\text { Syndactyly (other than minimal 2nd and } \\
\text { 3rd toes) }\end{array}$ \\
\hline & Large fontanelle & Anodontia/oligodontia & Microdontia & $\begin{array}{l}\text { Depressed } \\
\text { premaxillary } \\
\text { region }\end{array}$ & $\begin{array}{l}\text { Broad/bulbous } \\
\text { nasal tip }\end{array}$ & $\begin{array}{l}\text { Brittle hair/ } \\
\text { trichorrhexis } \\
\text { nodosa/pili torti }\end{array}$ & Irregular length or shape of toes \\
\hline & $\begin{array}{l}\text { Facies significantly } \\
\text { abnormal }\end{array}$ & Natal/neonatal teeth & Anodontia/oligodontia & $\begin{array}{l}\text { Midface } \\
\text { hypoplasia/flat/ } \\
\text { short midface }\end{array}$ & $\begin{array}{l}\text { Cleft soft palate/ } \\
\text { bifid uvula/ } \\
\text { submucous cleft }\end{array}$ & $\begin{array}{l}\text { Sparse/absent } \\
\text { scalp hair - } \\
\text { generalised }\end{array}$ & Other skull abnormality \\
\hline & Small face & Supernumerary teeth & Supernumerary teeth & $\begin{array}{l}\text { Micrognathia/ } \\
\text { agnathia/ } \\
\text { retrognathia }\end{array}$ & Microdontia & $\begin{array}{l}\text { High hairline - } \\
\text { front }\end{array}$ & Absent/small/hypoplastic carpals \\
\hline & Hypertelorism & Dental caries & $\begin{array}{l}\text { Other dental } \\
\text { abnormality }\end{array}$ & $\begin{array}{l}\text { Absent/decreased } \\
\text { eyebrows/lateral } \\
\text { thinning }\end{array}$ & $\begin{array}{l}\text { Abnormal tooth } \\
\text { position/ } \\
\text { malocclusion/open } \\
\text { bite }\end{array}$ & $\begin{array}{l}\text { Thin/ } \\
\text { hyperconvex/ } \\
\text { hypoplastic nails }\end{array}$ & Symphalangism \\
\hline
\end{tabular}


Table 3 Comparison of conditions associated with the simultaneous presence of microdontia and supernumery teeth along with taurodontism, microdontia, and dens invaginatus as well as distal symphalangism, hypoplastic carpal bones, microdontia, dental pulp stones, narrowed zygomatic arch. (Continued)

\begin{tabular}{|c|c|c|c|c|c|c|}
\hline $\begin{array}{l}\text { Prominent } \\
\text { supraorbital ridges }\end{array}$ & Low set ears & Simian creases & $\begin{array}{l}\text { Absent/decreased } \\
\text { lashes }\end{array}$ & $\begin{array}{l}\text { Supernumerary } \\
\text { teeth }\end{array}$ & $\begin{array}{l}\text { Dysplastic/ } \\
\text { grooved/thick/ } \\
\text { discoloured nails }\end{array}$ & Cone shaped epiphyses \\
\hline $\begin{array}{l}\text { Depressed } \\
\text { premaxillary } \\
\text { region }\end{array}$ & Scoliosis & Dislocated hip & $\begin{array}{l}\text { Everted/ } \\
\text { protruding lips }\end{array}$ & $\begin{array}{l}\text { Cleft/notched } \\
\text { tongue }\end{array}$ & Broad/bifid nails & Symphalangism \\
\hline $\begin{array}{l}\text { Midface } \\
\text { hypoplasia/flat/ } \\
\text { short midface }\end{array}$ & Bowed limbs & & $\begin{array}{l}\text { Tooth crown } \\
\text { shape } \\
\text { abnormality }\end{array}$ & $\begin{array}{l}\text { Hamartoma/other } \\
\text { tumours of the } \\
\text { mouth }\end{array}$ & Frontal bossing & $\begin{array}{l}\text { Cone-shaped epiphyses of middle } \\
\text { phalanges }\end{array}$ \\
\hline Prognathism & $\begin{array}{l}\text { Mental retardation of } \\
\text { any degree }\end{array}$ & & Microdontia & $\begin{array}{l}\text { Other abnormality } \\
\text { of tongue/ } \\
\text { gingivae/mucosa }\end{array}$ & High forehead & \\
\hline $\begin{array}{l}\text { Depressed nasal } \\
\text { bridge }\end{array}$ & $\begin{array}{l}\text { Boney sclerosis of any } \\
\text { type }\end{array}$ & & $\begin{array}{l}\text { Abnormal tooth } \\
\text { position/ } \\
\text { malocclusion/ } \\
\text { open bite }\end{array}$ & Low set ears & $\begin{array}{l}\text { Facies } \\
\text { significantly } \\
\text { abnormal }\end{array}$ & \\
\hline $\begin{array}{l}\text { Paramedian/lateral } \\
\text { cleft lip (uni/ } \\
\text { bilateral) }\end{array}$ & $\begin{array}{l}\text { Multiple fractures/ } \\
\text { increased boney fragility }\end{array}$ & & $\begin{array}{l}\text { Delayed eruption } \\
\text { of teeth }\end{array}$ & Tragus abnormal & Long face & \\
\hline $\begin{array}{l}\text { Cleft soft palate/ } \\
\text { bifid uvula/ } \\
\text { submucous cleft }\end{array}$ & $\begin{array}{l}\text { Enchondroma/ } \\
\text { radiolucencies - } \\
\text { localized }\end{array}$ & & $\begin{array}{l}\text { Anodontia/ } \\
\text { oligodontia }\end{array}$ & $\begin{array}{l}\text { Pectus excavatum } \\
\text { (funnel chest) }\end{array}$ & $\begin{array}{l}\text { Grooved/dimpled } \\
\text { chin }\end{array}$ & \\
\hline $\begin{array}{l}\text { High vaulted and } \\
\text { narrow palate }\end{array}$ & $\begin{array}{l}\text { Lytic/lucent lesions of } \\
\text { bone }\end{array}$ & & $\begin{array}{l}\text { Supernumerary } \\
\text { teeth }\end{array}$ & $\begin{array}{l}\text { Abnormally placed } \\
\text { nipples }\end{array}$ & $\begin{array}{l}\text { Micrognathia/ } \\
\text { agnathia/ } \\
\text { retrognathia }\end{array}$ & \\
\hline Microdontia & $\begin{array}{l}\text { Fibrous dysplasia of } \\
\text { bone }\end{array}$ & & & $\begin{array}{l}\text { Thoracolumbar } \\
\text { general kyphosis }\end{array}$ & $\begin{array}{l}\text { Medial flare of } \\
\text { eyebrows }\end{array}$ & \\
\hline $\begin{array}{l}\text { Developmental } \\
\text { defect of enamel }\end{array}$ & $\begin{array}{l}\text { Wide diaphyses } \\
\text { (undertubulation) }\end{array}$ & & & $\begin{array}{l}\text { Irregularities of } \\
\text { length/shape of } \\
\text { fingers }\end{array}$ & $\begin{array}{l}\text { Absent/decreased } \\
\text { eyebrows/lateral } \\
\text { thinning }\end{array}$ & \\
\hline $\begin{array}{l}\text { Tooth } \\
\text { discolouration }\end{array}$ & $\begin{array}{l}\text { Submetaphyseal } \\
\text { undermodelling/ } \\
\text { expansion }\end{array}$ & & & $\begin{array}{l}\text { Syndactyly of } \\
\text { fingers }\end{array}$ & $\begin{array}{l}\text { Absent/decreased } \\
\text { lashes }\end{array}$ & \\
\hline $\begin{array}{l}\text { Delayed eruption } \\
\text { of teeth }\end{array}$ & Thin cortex of diaphyses & & & $\begin{array}{l}\text { Polydactyly - } \\
\text { postaxial (ulnar)/ } \\
\text { type unspecified }\end{array}$ & Long/large nose & \\
\hline $\begin{array}{l}\text { Missing } \\
\text { permanent teeth/ } \\
\text { retained } \\
\text { deciduous teeth }\end{array}$ & Bowing of long bones & & & $\begin{array}{l}\text { Abnormal palmar } \\
\text { dermatoglyphics/ } \\
\text { skin creases }\end{array}$ & $\begin{array}{l}\text { Broad nasal } \\
\text { bridge (see } \\
\text { telecanthus) }\end{array}$ & \\
\hline $\begin{array}{l}\text { Supernumerary } \\
\text { teeth }\end{array}$ & $\begin{array}{l}\text { Cartilage tongues of } \\
\text { metaphyses - localized }\end{array}$ & & & $\begin{array}{l}\text { Polydactyly of feet } \\
\text { - postaxial/type } \\
\text { unspecified }\end{array}$ & High nasal bridge & \\
\hline $\begin{array}{l}\text { Dental cysts/ } \\
\text { tumours }\end{array}$ & $\begin{array}{l}\text { Hyperostosis/thickened/ } \\
\text { sclerotic calvarium }\end{array}$ & & & $\begin{array}{l}\text { Syndactyly (other } \\
\text { than minimal 2nd } \\
\text { and 3rd toes) }\end{array}$ & $\begin{array}{l}\text { Broad/bulbous } \\
\text { nasal tip }\end{array}$ & \\
\hline
\end{tabular}


Table 3 Comparison of conditions associated with the simultaneous presence of microdontia and supernumery teeth along with taurodontism, microdontia, and dens invaginatus as well as distal symphalangism, hypoplastic carpal bones, microdontia, dental pulp stones, narrowed zygomatic arch. (Continued)

\begin{tabular}{|c|c|c|c|}
\hline $\begin{array}{l}\text { Deafness - } \\
\text { conductive }\end{array}$ & $\begin{array}{l}\text { Absent/abnormal } \\
\text { sinuses }\end{array}$ & $\begin{array}{l}\text { Cranial nerve/ } \\
\text { nuclei }\end{array}$ & $\begin{array}{l}\text { Hypoplastic/small } \\
\text { nostrils }\end{array}$ \\
\hline $\begin{array}{l}\text { Other hearing } \\
\text { abnormality }\end{array}$ & Wormian bones & $\begin{array}{l}\text { Mental retardation } \\
\text { - moderate/severe }\end{array}$ & $\begin{array}{l}\text { Abnormal } \\
\text { columella }\end{array}$ \\
\hline $\begin{array}{l}\text { Narrow/sloping } \\
\text { shoulder/ } \\
\text { hypermobile } \\
\text { shoulders }\end{array}$ & $\begin{array}{l}\text { Sclerotic/hyperostotic } \\
\text { facial bones }\end{array}$ & Hypotonia & Thin lips \\
\hline $\begin{array}{l}\text { Pectus excavatum } \\
\text { (funnel chest) }\end{array}$ & Other skull abnormality & $\begin{array}{l}\text { Movement } \\
\text { disorder - } \\
\text { dystonia/chorea/ } \\
\text { tremor/spasm }\end{array}$ & Long philtrum \\
\hline Bell-shaped chest & $\begin{array}{l}\text { Hyperostotic/wide } \\
\text { clavicle }\end{array}$ & EEG abnormality & $\begin{array}{l}\text { Deeply grooved } \\
\text { philtrum }\end{array}$ \\
\hline $\begin{array}{l}\text { Thoracolumbar } \\
\text { general kyphosis }\end{array}$ & $\begin{array}{l}\text { Abnormal rib structure } \\
\text { including fusion }\end{array}$ & Short sternum & Microdontia \\
\hline $\begin{array}{l}\text { Gibbus/localised } \\
\text { kyphosis }\end{array}$ & Widened ribs & & $\begin{array}{l}\text { Abnormal tooth } \\
\text { position/ } \\
\text { malocclusion/ } \\
\text { open bite }\end{array}$ \\
\hline Scoliosis & $\begin{array}{l}\text { Irregular shape of pubic } \\
\text { and ischial bones }\end{array}$ & & $\begin{array}{l}\text { Supernumerary } \\
\text { teeth }\end{array}$ \\
\hline $\begin{array}{l}\text { Hyperextensible/ } \\
\text { hypermobile joints }\end{array}$ & $\begin{array}{l}\text { Absent/hypoplastic/ } \\
\text { short femur }\end{array}$ & & $\begin{array}{l}\text { Anteverted/ } \\
\text { prominent/bat } \\
\text { ears }\end{array}$ \\
\hline Small hand & $\begin{array}{l}\text { Femora short/ } \\
\text { deformed/bowed }\end{array}$ & & Long/large ear \\
\hline Brachydactyly & Other abnormal femur & & $\begin{array}{l}\text { Pectus carinatum } \\
\text { (pigeon chest) }\end{array}$ \\
\hline $\begin{array}{l}\text { Seizures of any } \\
\text { type }\end{array}$ & Bow legs - genu varum & & $\begin{array}{l}\text { Thoracolumbar } \\
\text { general kyphosis }\end{array}$ \\
\hline Hypotonia & & & Scoliosis \\
\hline $\begin{array}{l}\text { Imperforate anus/ } \\
\text { anal stenosis }\end{array}$ & & & $\begin{array}{l}\text { Hyperextensible/ } \\
\text { hypermobile } \\
\text { joints }\end{array}$ \\
\hline $\begin{array}{l}\text { Horseshoe/fused/ } \\
\text { ectopic kidneys }\end{array}$ & & & Small hand \\
\hline $\begin{array}{l}\text { Hypospadias/ } \\
\text { epispadias }\end{array}$ & & & Brachydactyly \\
\hline $\begin{array}{l}\text { Undescended/ } \\
\text { ectopic testes }\end{array}$ & & & $\begin{array}{l}\text { Clinodactyly of } \\
5 \text { th finger }\end{array}$ \\
\hline Wilms tumour & & & $\begin{array}{l}\text { Terminal } \\
\text { hypoplasia fingers }\end{array}$ \\
\hline
\end{tabular}


Table 3 Comparison of conditions associated with the simultaneous presence of microdontia and supernumery teeth along with taurodontism, microdontia, and dens invaginatus as well as distal symphalangism, hypoplastic carpal bones, microdontia, dental pulp stones, narrowed zygomatic arch. (Continued)

\begin{tabular}{|c|c|}
\hline $\begin{array}{l}\text { Delayed skeletal } \\
\text { maturation }\end{array}$ & $\begin{array}{l}\text { Spindle shaped/ } \\
\text { tapered fingers }\end{array}$ \\
\hline $\begin{array}{l}\text { Poorly ossified } \\
\text { calvarium/Soft } \\
\text { skull }\end{array}$ & $\begin{array}{l}\text { Ulnar deviation of } \\
\text { fingers }\end{array}$ \\
\hline $\begin{array}{l}\text { Absent/abnormal } \\
\text { sinuses }\end{array}$ & $\begin{array}{l}\text { Other hand } \\
\text { abnormality }\end{array}$ \\
\hline Wormian bones & $\begin{array}{l}\text { Mental } \\
\text { retardation of any } \\
\text { degree }\end{array}$ \\
\hline $\begin{array}{l}\text { Platybasia/basilar } \\
\text { impression }\end{array}$ & $\begin{array}{l}\text { Abnormal } \\
\text { cardiovascular } \\
\text { structure/function }\end{array}$ \\
\hline $\begin{array}{l}\text { Enlarged foramen } \\
\text { magnum }\end{array}$ & $\begin{array}{l}\text { Winged/other } \\
\text { abnormal scapula } \\
\text { (See Shoulder) }\end{array}$ \\
\hline $\begin{array}{l}\text { Small/absent } \\
\text { scapula }\end{array}$ & Coxa vara \\
\hline $\begin{array}{l}\text { Winged/other } \\
\text { abnormal scapula } \\
\text { (See Shoulder) }\end{array}$ & $\begin{array}{l}\text { Cone shaped } \\
\text { epiphyses }\end{array}$ \\
\hline $\begin{array}{l}\text { Absent/ } \\
\text { hypoplastic } \\
\text { clavicles }\end{array}$ & $\begin{array}{l}\text { Small femoral } \\
\text { head epiphyses }\end{array}$ \\
\hline $\begin{array}{l}\text { Pseudarthrosis of } \\
\text { clavicle }\end{array}$ & $\begin{array}{l}\text { Flat femoral head } \\
\text { epiphyses }\end{array}$ \\
\hline $\begin{array}{l}\text { Short ribs } \\
\text { (circumferential) }\end{array}$ & $\begin{array}{l}\text { Deformed/ } \\
\text { irregular femoral } \\
\text { head epiphyses }\end{array}$ \\
\hline $\begin{array}{l}\text { Under-/unossified } \\
\text { sternum }\end{array}$ & $\begin{array}{l}\text { Broad femoral } \\
\text { neck }\end{array}$ \\
\hline $\begin{array}{l}\text { Hypoplastic/ } \\
\text { absent ribs }\end{array}$ & $\begin{array}{l}\text { Cone-shaped } \\
\text { epiphyses of } \\
\text { proximal } \\
\text { phalanges }\end{array}$ \\
\hline $\begin{array}{l}\text { Dorsal wedging of } \\
\text { vertebral bodies }\end{array}$ & $\begin{array}{l}\text { Some phalanges } \\
\text { short and } \\
\text { deformed }\end{array}$ \\
\hline $\begin{array}{l}\text { Narrow/trapezoid } \\
\text { iliac wings (lack of } \\
\text { flare) }\end{array}$ & $\begin{array}{l}\text { Cone-shaped } \\
\text { epiphyses of } \\
\text { middle phalanges }\end{array}$ \\
\hline $\begin{array}{l}\text { Horizontal/flat } \\
\text { acetabular roof }\end{array}$ & $\begin{array}{l}\text { Cone-shaped } \\
\text { epiphyses of } \\
\text { distal phalanges }\end{array}$ \\
\hline
\end{tabular}


Table 3 Comparison of conditions associated with the simultaneous presence of microdontia and supernumery teeth along with taurodontism, microdontia, and dens invaginatus as well as distal symphalangism, hypoplastic carpal bones, microdontia, dental pulp stones, narrowed zygomatic arch. (Continued)

Delayed

ossification of

pubic and ischia

bones

Open pubic

symphysis in

adults

Coxa valga

Coxa vara

Dislocated hip

Cone shaped

epiphyses

Fibulae a-/

hypoplastic/

under-/unossified

Cone-shaped

epiphyses of

proximal

phalanges

Cone-shaped

epiphyses of

middle phalanges

All middle

phalanges short

deformed

Cone-shaped

epiphyses of distal

phalange

All distal

phalanges short/

deformed 
difficult delivery, it was insignificant and neither microdontia nor mesiodens has been reported in the literature.

The prevalence of mesiodens varies between 0.09 and $2.05 \%$ in different studies. In permanent dentition, a 0.15 to $3.8 \%$ incidence of mesiodens has been reported [19]. Erupted supernumerary teeth in the mandible are rare, is about $0.01 \%$ which indicated marked low value [20]. Supernumerary teeth in the mandible anterior region in this case is fully erupted which is unusual.

Sexual dimorphism is reported by most authors with males being more commonly affected. Hogstrum and Andersson [21] reported a 2:1 ratio of sex distribution. A study of supernumerary teeth in Asian school children found a greater male to female distribution of 6.5:1 for Hong Kong children [22] which indicates that supernumery teeth is more common in males than females which is consistent in our case.

Non-syndromic multiple supernumerary teeth occur most frequently in the mandible region especially premolar region followed by molar and anterior region [9]. Few cases of non-syndrome multiple supernumery teeth have been reported $[23,24]$ however in the present case nonsyndromic single supernumerary tooth was observed in the mandibular anterior region.

Evidence regarding etiology of mesiodens indicates that genetic susceptibility together with environmental factors might increase the activity of dental lamina leading to formation of the extra tooth/teeth [19]. A number of theories have been proposed as regards the causes of the occurrence of supernumerary teeth: 1$]$ Atavism theory $[8,24,25]$ 2] Independent hyperactivity of the dental lamina $[24,25]$ and 3] Dichotomy of the tooth bud are also suggested as a possible etiological factors $[8,25]$. However, none of these theories alone offers a sufficient explanation for this phenomenon.

Since mesiodens may interfere with normal occlusal development, in the present case an early diagnosis could have prevented the lower diastema formation. Early diagnosis and treatment of patients with supernumerary teeth are important to prevent or minimize complications.

As the patient did not show any abnormal systemic manifestations, all the syndrome associated with the dental anomalies were ruled out. The simultaneous presence of supernumerary teeth and the generalized microdontia is very rare. To our knowledge, this is the first such case of non-syndromic occurance of true generalized microdontia in association with mandibular mesiodens. Such unusual nature of dental anomaly has not been reported so far in the literature.

\section{Conclusion}

The dental finding seen in this case is certainly rare. The case is also sporadic, with no positive family history. The wide variation in clinical manifestations in cases of non- syndromic occurrence of dental anomalies is challenging and is an area for further research. Mesiodens are familiar to pediatric dentists and orthodontists as one of the more common anomalies to affect the developing dentition and it demands a multidisciplinary assessment.

\section{Consent}

Written informed consent was obtained from the patient for publication of this Case report and any accompanying images. A copy of the written consent is available for review by the Editor-in-Chief of this journal.

\section{Acknowledgements}

The authors also wish to thank the patient and their family for their contribution to this article. Written consent for publication was obtained from the patient's parent.

\section{Authors' contributions}

SB and SK drafted the manuscript paper, analysed the patient's history and contributed to the writing of the final version as well as extracted the mesiodens. Each author reviewed the paper for content and contributed to the writing of the manuscript. All authors approved the final report.

\section{Competing interests}

The authors declare that they have no competing interests.

Received: 25 May 2011 Accepted: 28 October 2011

Published: 28 October 2011

\section{References}

1. Laundau S: International dictionary of medicine and biology. New York: John Wiley \& Sons; 1 1986, 1717.

2. Boyle PE: Kronfeld's Histopathology of the Teeth and their Surrounding Structures. Philadelphia: Lea\& Febiger; 3 1955, 14.

3. Shafer WG, Hine MK, Levy BM: A Textbook of Oral Pathology. Philadelphia: W. B. Saunders Co; 1 1958, 26.

4. Ufomata D: Microdontia of a mandibular second premolar. Oral Surg Oral Med Oral Pathol 1988, 65:637-8.

5. Van der waal I, Van der kwast WAM: Developmental anomalies and eruption disturbances and some acquired disorders of the teeth. Oral pathology Chicago: Quintessence Publishing Co. Inc; 1988, 114.

6. Opinya GN, Kaimenyi JT, Meme JS: Oral findings in Fanconi's anemia: a case report. J Periodontol 1988, 59:461-463.

7. Schulze C: Developmental abnormalities of the teeth and jaws. In Thoma's Oral Pathology. Edited by: Gorlin RJ, Goldman HM. St Louis: Mosby; 1970:112-22.

8. Rajab LD, Hamdan MA: Supernumerary teeth: review of the literature and a survey of 152 cases. Int J Paediatr Dent 2002, 12:244-54.

9. Bhat M: Supplemental mandibular central incisor. J Indian Soc Pedod Prev Dent 2006, 24(Suppl 2):s20-s23

10. Tanaka S, Murakami Y, Fukami M, Nakano K, Fujisawa S, Miyoshi S: A rare case of bilateral supernumerary teeth in the mandibular incisors. Br Dent J 1998, 185:386-8.

11. Fukuta $Y$, Totsuka M, Takeda $Y$, Yamamoto H: Supernumerary teeth with eumorphism in the lower incisor region:a report of five cases and review of the literature. J Oral Sci 1999, 41:199-202.

12. Alencar M, Duarte D, Cury P, Bonecker M: Lower mesiodens: report of an unusual case. J Clin Pediatr Dent 2005, 29:353-356.

13. Yokose T, Sakamoto T, Sueishi K, Yatabe K, Tsujino K, Kubo S, Yakushiji M, Yamaguchi H: Two cases with supernumerary teeth in lower incisor region. Bull Tokyo Dent Coll 2006, 47:19-23.

14. Grga D, Dzeletovic B: Supernumerary Tooth in Lower Incisor Region: A Case Report. Serbian Dental Journal 2010, 57:220-222.

15. Zhu JF, Marcushamer M, King DL, Henry RJ: Supernumerary and Congenitally absent teeth: a literature review. J Clin Pediat Dentist 1996, 20:87-95. 
16. Thesleff I: Genetic basis of development of dental defects. Acta Odontol Scand 2000, 58:191-4.

17. Osborn JW, Ten Cate AR: Advanced dental histology: dental practitioner. Handbook no, 6. Bristol: John Wright \& Sons; 3 1976, 24-51.

18. Alexandersen V, Nielsen OV: Generalized microdontia probably associated with intrauterine growth retardation in a medieval skeleton. American Journal of Physical Anthropology 1970, 33:389-401.

19. Meighani G, Pakdaman A: Diagnosis and Management of Supernumerary (Mesiodens): A Review of the Literature. Journal of Dentistry 2010, 7:41-49.

20. Stafne EC: Supernumerary teeth. Dental Cosmos 1932, 74:653-659.

21. Hogstrom A, Andersson L: Complications related to surgical removal of anterior supernumerary teeth in children. ASDC J Dent Child 1987, 54:341-3.

22. Davis PJ: Hypodontia and hyperodontia of permanent teeth in Hong Kong school children. Community Dentistry and Oral Epidemiology 1987, 15:218-20

23. Srivatsan $\mathrm{P}$, Aravindha Babu N: Mesiodens with an unusual morphology and multiple impacted supernumerary teeth in a non-syndromic patient. Indian J Dent Res 2007, 18:138-40.

24. Sivapathasundharam B, Einstein A: Non-syndromic multiple supernumerary teeth: Report of a case with 14 supplemental teeth. Indian J Dent Res 2007, 18:144.

25. Stellzig A, Basdra EK, Komposch G: Mesiodentes: incidence, morphology, etiology. J Orofac Orthopaedic 1997, 58:144-153.

doi:10.1186/1746-160X-7-19

Cite this article as: Bargale and Kiran: Non-syndromic occurrence of true generalized microdontia with mandibular mesiodens - a rare case. Head \& Face Medicine 2011 7:19.

\section{Submit your next manuscript to BioMed Central and take full advantage of:}

- Convenient online submission

- Thorough peer review

- No space constraints or color figure charges

- Immediate publication on acceptance

- Inclusion in PubMed, CAS, Scopus and Google Scholar

- Research which is freely available for redistribution

Submit your manuscript at www.biomedcentral.com/submit 